\title{
On the Channel Capacity of Multilevel Modulation Schemes with Coherent Detection
}

\author{
Ivan B. Djordjevic ${ }^{1 \mathrm{a}}$, Lei Xu ${ }^{\mathrm{b}}$, Ting Wang ${ }^{\mathrm{b}}$ \\ ${ }^{a}$ University of Arizona, Department of Electrical \& Computer Engineering, Tucson, AZ, USA 85721 \\ ${ }^{b}$ NEC Laboratories America, Princeton, NJ, USA 08540
}

\begin{abstract}
We describe a method to determine the channel capacity of an arbitrary multilevel modulation scheme by modeling the fiber-optic channel as a dynamical nonlinear ISI channel with memory. We also propose a multilevel turbo-equalization scheme that is able closely to approach the channel capacity. We show that with this scheme we are able straightforwardly to upgrade currently installed $10 \mathrm{~Gb} / \mathrm{s}$ optical transmission systems to $100 \mathrm{~Gb} / \mathrm{s}$, and even with small memory assumption we are able to achieve $100 \mathrm{~Gb} / \mathrm{s}$ per DWDM channel transmission over $9600 \mathrm{~km}$.
\end{abstract}

Keywords: Fiber-optics communications, channel capacity, modulation, forward error correction (FEC), low-density parity-check (LDPC) codes

\section{INTRODUCTION}

As data rates transmitted over the optical fiber increase, a fundamental question about physical limitations of optical fiber arises. The problem of determining capacity of optical transmission systems has been addressed by numerous researchers ${ }^{1-8}$. The main approach, until recently, was to consider amplified spontaneous emission (ASE) noise as a predominant effect and to observe the fiber nonlinearities as the perturbation of linear case ${ }^{1,2}$ or as the multiplicative noise ${ }^{3}$. Essiambre at al. ${ }^{1}$ calculate the channel capacity for nonbinary modulation formats by assuming that the optical channel is discrete and memoryless. The nonlinear nature of the propagation of light in optical fiber systems plays a crucial role in limiting the capacity and makes these limits difficult to calculate.

In our recent publications ${ }^{6,7}$ we proposed a method to determine the achievable information rates (lower bounds on channel capacity) for high-speed optical transmission using the finite state machine approach, when the combined effects of ASE noise, Kerr nonlinearity, stimulated Raman scattering, chromatic dispersion, polarization mode dispersion (PMD) and (optical/electrical) filtering are taken into account. In these publications, the channel capacity study has been performed for binary modulation formats only.

In this paper, we describe a method to determine the independent identically distributed (IID) channel capacity of arbitrary multilevel modulation scheme by modeling the fiber-optic channel as dynamical nonlinear intersymbol interference (ISI) channel with memory. Moreover, we describe a coding scheme that is able is able closely to approach the IID channel capacity. This scheme is based on multilevel turbo equalization and coded-modulation, and employs the best known low-density parity check (LDPC) codes as channel codes. We show that with this scheme we are able straightforwardly to upgrade currently installed $10 \mathrm{~Gb} / \mathrm{s}$ optical transmission systems to $100 \mathrm{~Gb} / \mathrm{s}$. We show that implementing the proposed scheme even with small memory assumption we are able to achieve $100 \mathrm{~Gb} / \mathrm{s}$ per DWDM channel transmission over $9600 \mathrm{~km}$.

The paper is organized as follows. In Section 2 we first provide channel capacity preliminaries. We then describe how to determine the channel capacity of fiber-optics communication systems for arbitrary multilevel modulation format. The LDPC-coded turbo equalization scheme, based on multilevel maximum a posteriori probability (MAP) equalizer, implemented using BCJR algorithm ${ }^{9}$, is described in Section 3. In same section we study the efficiency of the LDPCcoded turbo equalizer in suppression of fiber nonlinearities. Finally, in Section 4, some important concluding remarks are given.

1ivan@ece.arizona.edu; phone 1520 626-5119; fax 1520 626-3144; www.ece.arizona.edu/ ivan/

Optical Transmission Systems, Switching, and Subsystems VII, edited by Dominique Chiaroni,

Proc. of SPIE-OSA-IEEE Asia Communications and Photonics, SPIE Vol. 7632, 76320W

(C) 2009 SPIE-OSA-IEEE • CCC code: 0277-786X/09/\$18 • doi: 10.1117/12.851122 


\section{CHANNEL CAPACITY OF MULTILEVEL MODULATION SCHEMES}

\subsection{Channel Capacity Preliminaries}

The channel code considers whole transmission system as a discrete channel, in which the sizes of input and output alphabets are finite. Two examples of such channel are shown in Fig. 1. In Fig. 1(a) we show an example a discrete memoryless channel (DMC), which is characterized by channel (transition) probabilities. Let $X=\left\{x_{0}, x_{1}, \ldots, x_{I-1}\right\}$ denote the channel input alphabet, and $Y=\left\{y_{0}, y_{1}, \ldots, y_{J-1}\right\}$ denote the channel output alphabet. This channel is completely characterized by the following set of transition probabilities:

$$
p\left(y_{j} \mid x_{i}\right)=P\left(Y=y_{j} \mid X=x_{i}\right), 0 \leq p\left(y_{j} \mid x_{i}\right) \leq 1, i \in\{0,1, \ldots, I-1\}, j \in\{0,1, \ldots, J-1\},
$$

where $I$ and $J$ denote the sizes of input and output alphabets, respectively. The transition probability $p\left(y_{j} \mid x_{i}\right)$ represents the conditional probability that channel output $Y=y_{j}$ given the channel input $X=x_{i}$. The channel introduces the errors, and if $j \neq i$ the corresponding $p\left(y_{j} \mid x_{i}\right)$ represents the conditional probability of error, while for $j=i$ it represents the conditional probability of correct reception. For $I=J$, the average symbol error probability is defined as the probability that output random variable $Y_{j}$ is different from input random variable $X_{i}$, with averaging being performed for all $j \neq i$ :

$$
P_{e}=\sum_{i=0}^{I-1} p\left(x_{i}\right) \sum_{j=0, j \neq i}^{J-1} p\left(y_{j} \mid x_{i}\right)
$$

where the inputs are selected from the following distribution $\left\{p\left(x_{i}\right)=P\left(X=x_{i}\right) ; i=0,1, . ., I-1\right\}$, with $p\left(x_{i}\right)$ being known as a priori probability of input symbol $x_{i}$. The corresponding probabilities of output symbols can be calculated by:

$$
p\left(y_{j}\right)=\sum_{i=0}^{I-1} P\left(Y=y_{j} \mid X=x_{i}\right) P\left(X=x_{i}\right)=\sum_{i=0}^{I-1} p\left(y_{j} \mid x_{i}\right) p\left(x_{i}\right) ; j=0,1, \ldots, J-1 .
$$

The decision rule that minimizes average symbol error probability (2), denoted as $D\left(y_{j}\right)=x^{*}$, is known as maximum a posteriori (MAP) rule, and can be formulated as follows:

$$
D\left(y_{j}\right)=x^{*}: \quad P\left(x^{*} \mid y_{j}\right) \geq P\left(x_{i} \mid y_{j}\right), \quad i=0,1, \ldots, I-1
$$

Therefore, the symbol error probability $P_{e}$ will be minimal when to every output symbol $y_{j}$ the input symbol $x^{*}$ is assigned having largest a posteriori probability $P\left(x^{*} \mid y_{j}\right)$. By using the Bayes' rule the equation (4) can be re-written as

$$
D\left(y_{j}\right)=x^{*}: \frac{P\left(y_{j} \mid x^{*}\right) P\left(x^{*}\right)}{P\left(y_{j}\right)} \geq \frac{P\left(y_{j} \mid x_{i}\right) P\left(x_{i}\right)}{P\left(y_{j}\right)}, \quad i=0,1, \ldots, I-1
$$

If all input symbols are equally likely $P\left(x_{i}\right)=1 / I(i=0, \ldots, I-1)$, the corresponding decision rule is known as maximumlikelihood (ML) decision rule:

$$
D\left(y_{j}\right)=x^{*}: \quad P\left(y_{j} \mid x^{*}\right) \geq P\left(y_{j} \mid x_{i}\right), \quad i=0,1, \ldots, I-1
$$

In Fig. 1(b) we show a discrete channel model with memory ${ }^{7}$, which is more suitable for optical communications for binary transmission, because the optical channel is essentially the channel with memory. We assume that the optical channel has the memory equal to $2 m+1$, with $2 m$ being the number of bits that influence the observed bit from both sides. This dynamical trellis is uniquely defined by the set of previous state, the next state, in addition to the channel output. The state (the bit-pattern configuration) in the trellis is defined as $\boldsymbol{s}_{j}=\left(x_{j-m}, x_{j-m+1}, . ., x_{j}, x_{j+1}, \ldots, x_{j+m}\right)=\boldsymbol{x}[j-m, j+m]$, where $x_{k} \in \boldsymbol{X}=\{0,1\}$. An example trellis of memory $2 m+1=5$ is shown in Fig. 1(b). The trellis has $2^{5}=32$ states $\left(\boldsymbol{s}_{0}, \boldsymbol{s}_{1}, \ldots, \boldsymbol{s}_{31}\right)$, each of which corresponds to a different 5-bit pattern. For the complete description of the trellis, the transition probability density functions (PDFs) $p\left(y_{j} \mid x_{j}\right)=p\left(y_{j} \mid \mathbf{s}\right), \boldsymbol{s} \in \boldsymbol{S}$ can be determined from collected histograms, where $y_{j}$ represents the sample that corresponds to the transmitted bit $x_{j}$, and $\boldsymbol{S}$ is the set of states in the trellis. 


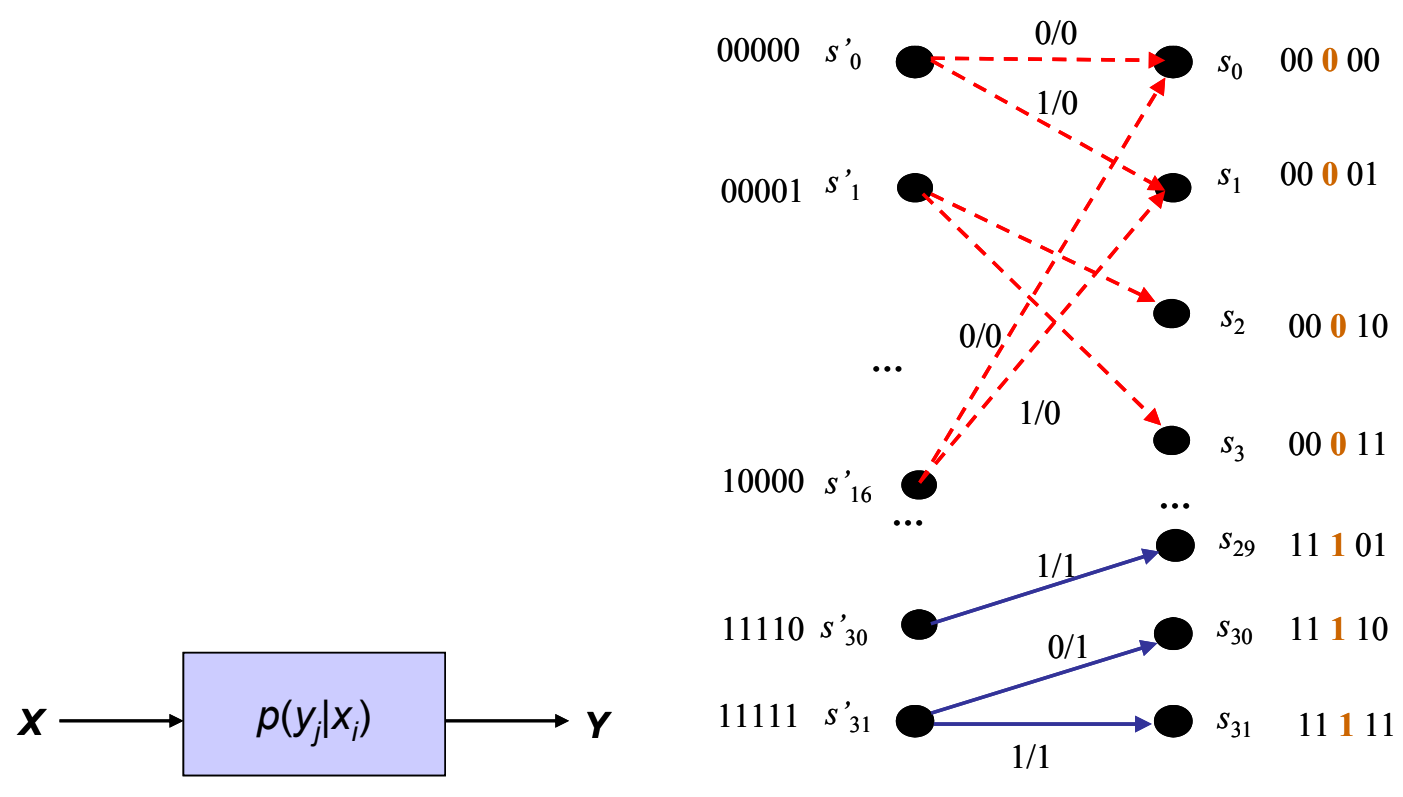

(a)

(b)

Figure 1. Two examples of discrete channels: (a) discrete memoryless channel (DMC), and (b) discrete channel with memory for binary transmission described as dynamic trellis.

One important figure of merit for DMCs is the amount information conveyed by the channel, which is known as the mutual information and it is defined as ${ }^{11,12}$

$$
I(\boldsymbol{X} ; \boldsymbol{Y})=H(\boldsymbol{X})-H(\boldsymbol{X} \mid \boldsymbol{Y})=\sum_{i=0}^{I-1} p\left(x_{i}\right) \log _{2}\left[\frac{1}{p\left(x_{i}\right)}\right]-\sum_{j=0}^{J-1} p\left(y_{j}\right) \sum_{i=0}^{I-1} p\left(x_{i} \mid y_{j}\right) \log _{2}\left[\frac{1}{p\left(x_{i} \mid y_{j}\right)}\right],
$$

where $H(\boldsymbol{X})$ denotes the uncertainty about the channel input before observing the channel output, also known as entropy; while $H(\boldsymbol{X} \mid \boldsymbol{Y})$ denotes the conditional entropy or the amount of uncertainty remaining about the channel input after the channel output has been received. Therefore, the mutual information represents the amount of information (per symbol) that is conveyed by the channel, that is the uncertainty about the channel input that is resolved by observing the channel output. The mutual information can be interpreted by means of Venn diagram shown in Fig. 2(a). The left circle represents the entropy of channel input, the right circle represents the entropy of channel output, and the mutual information is obtained in intersection of these two circles. Another interpretation due to Ingels ${ }^{12}$ is shown in Fig. 2(b). The mutual information, i.e. the information conveyed by the channel, is obtained as output information minus information lost in the channel.

It is clear from equation (7) that mutual information is independent on the channel, and someone may try to maximize the information conveyed by the channel, to obtain the so called channel capacity:

$$
C=\max _{\left\{p\left(x_{i}\right)\right\}} I(\boldsymbol{X} ; \boldsymbol{Y}) ; \text { subject to : } p\left(x_{i}\right) \geq 0 \quad \sum_{i=0}^{I-1} p\left(x_{i}\right)=1 .
$$

Now we have built enough knowledge to formulate the channel coding theorem ${ }^{11,12}$ :

Let a discrete memoryless source with an alphabet $\boldsymbol{S}$ have entropy $H(\boldsymbol{S})$ and emit the symbols every $T_{\mathrm{s}}$ seconds. Let a discrete memoryless channel have capacity $C$ and be used once in $T_{c}$ seconds. Then, if

$$
H(\boldsymbol{S}) / T_{\mathrm{s}} \leq C / T_{\mathrm{c}}
$$


there exists a coding scheme for which the source output can be transmitted over the channel and reconstructed with an arbitrary small probability of error. The parameter $H(\boldsymbol{S}) / T_{\mathrm{s}}$ is related to the average information rate, while the parameter $C / T_{\mathrm{c}}$ is related to the channel capacity per unit time. For binary symmetric channel $(I=J=2)$ the inequality (9) simply becomes

$$
R \leq C,
$$

where $R$ is the code rate introduced above.
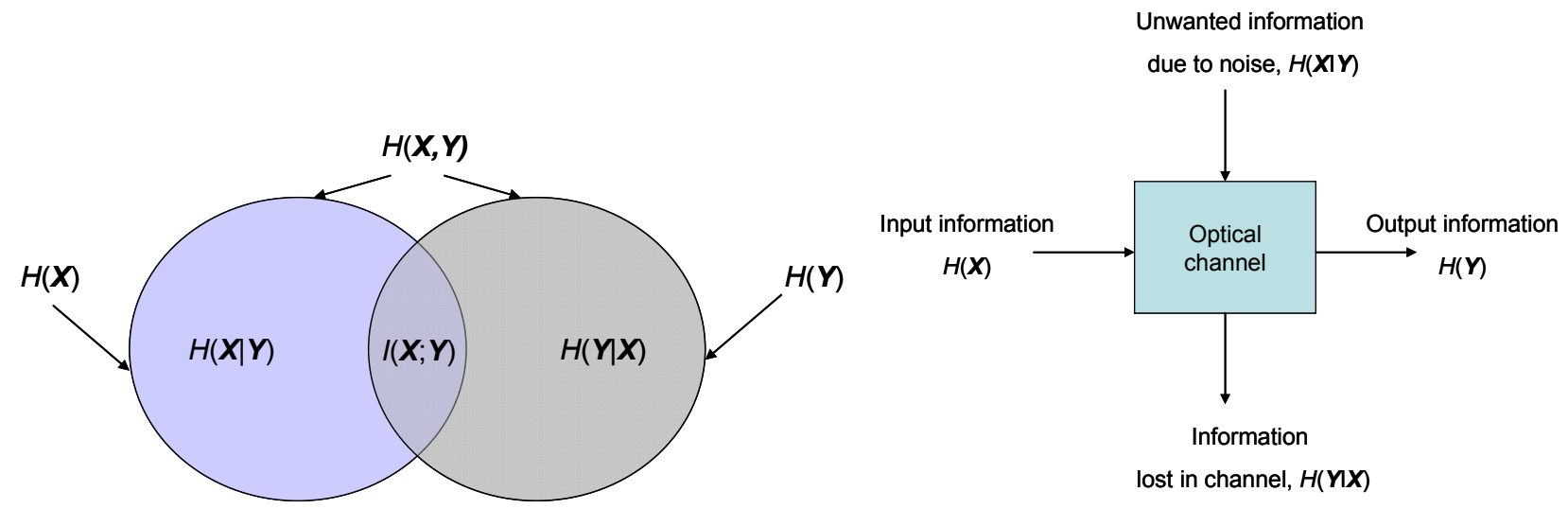

(a)

(b)

Figure 2. Interpretation of the mutual information: (a) using Venn diagrams, and (b) using the approach due to Ingels.

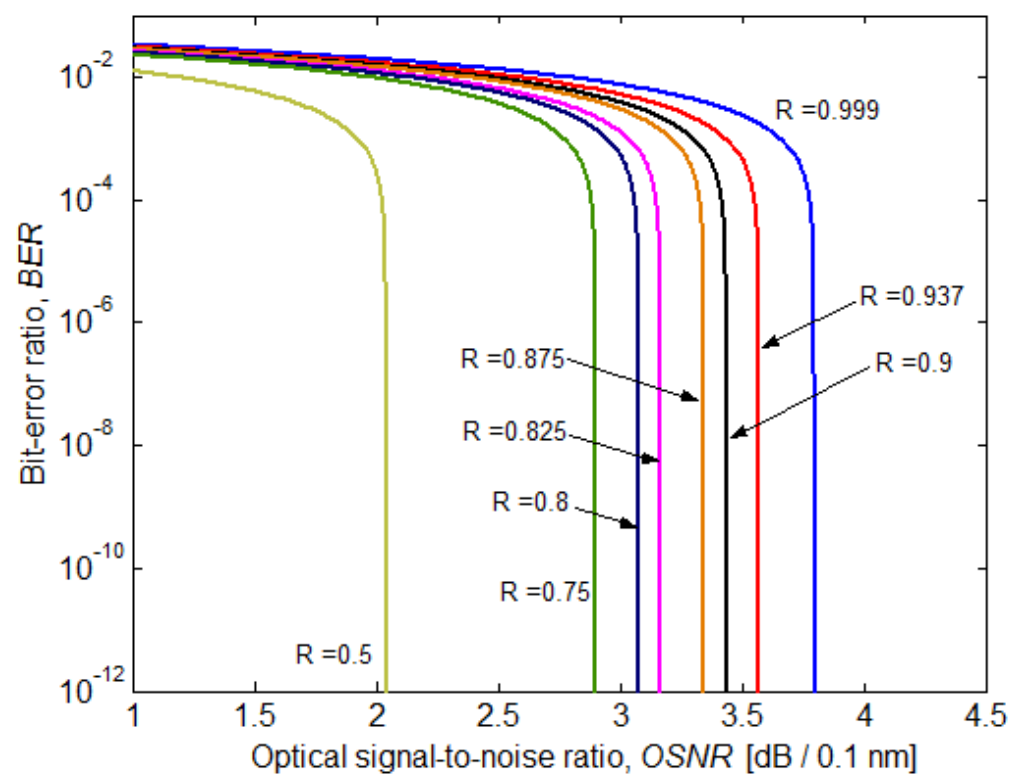

Figure 3. Minimum BER against optical SNR for different code rate values (for BPSK at $40 \mathrm{~Gb} / \mathrm{s}$ ).

Another very much important theorem is the Shannon's third theorem, also known as the information capacity theorem, and can be formulated as follows ${ }^{11,12}$ :

The information capacity of a continuous channel of bandwidth $B \mathrm{~Hz}$, perturbed by AWGN of PSD $N_{0} / 2$ and limited in bandwidth $B$, is given by 


$$
C=B \log _{2}\left(1+\frac{P}{N_{0} B}\right)[\mathrm{bits} / \mathrm{s}],
$$

where $P$ is the average transmitted power. This theorem represents remarkable result of information theory, because it connects all important system parameters (transmitted power, channel bandwidth and noise power spectral density) in only one formula. What is also interesting is that LDPC codes can approach the Shannon's limit within $0.0045 \mathrm{~dB}^{13}$. By using the Eq. (11) and Fano's inequality ${ }^{11}$

$$
H(\boldsymbol{X} \mid \boldsymbol{Y}) \leq H\left(P_{e}\right)+P_{e} \log _{2}(I-1), \quad H\left(P_{e}\right)=-P_{e} \log _{2} P_{e}-\left(1-P_{e}\right) \log _{2}\left(1-P_{e}\right)
$$

for amplified spontaneous emission (ASE) noise dominated scenario and binary phase-shift keying (BPSK) at $40 \mathrm{~Gb} / \mathrm{s}$ in Fig. 3 we report the minimum BERs against optical SNR for different code rates.

\subsection{Calculation of Information Capacity of Multilevel Modulation Schemes by Forward Recursion of BCJR Algorithm}

Here we address the problem of calculating of channel capacity of multilevel modulation schemes for an IID information source, in literature also known as the achievable information rate (see Djordjevic et al. ${ }^{6,7}$ and references therein). The IID channel capacity represents a lower bound on channel capacity. To calculate the IID channel capacity, we model the whole transmission system as the dynamical ISI channel, in which $m$ previous and next $m$ symbols influence the observed symbol. The optical communication system is characterized by the conditional probability density function (PDF) of the output complex vector of samples $\boldsymbol{y}=\left(y_{1}, \ldots, y_{n}, \ldots\right)$, where $y_{i}=\left(\operatorname{Re}\left\{y_{i}\right\}, \operatorname{Im}\left\{y_{i}\right\}\right) \in \boldsymbol{Y}$, given the source sequence $\boldsymbol{x}=\left(x_{1}, \ldots, x_{n}, \ldots\right), x_{i} \in \boldsymbol{X}=\{0,1, \ldots, M-1\}$. The set $\boldsymbol{X}$ represents the set of indices of constellation points in corresponding $M$ ary two-dimensional signal constellation diagram (such as $M$-ary phase-shift keying (PSK), $M$-ary quadrature-amplitude modulation (QAM) or $M$-ary polarization-shift keying (PolSK)), while $\boldsymbol{Y}$ represents the set of all possible channel outputs. The $\operatorname{Re}\left\{y_{i}\right\}$ corresponds to the in-phase channel sample, and the $\operatorname{Im}\left\{y_{i}\right\}$ represents the quadrature channel sample. An example of dynamical channel description by means of trellis diagram is shown in Fig. 4(a) for 4-level modulation formats, such as QPSK. This dynamical trellis is uniquely defined by the following triplet: the previous state, the next state, and the channel output. The state in the trellis is defined as $\boldsymbol{s}_{j}=\left(x_{j-m}, x_{j-m+1}, . ., x_{j}, x_{j+1}, \ldots, x_{j+m}\right)=\boldsymbol{x}[j-m, j+m]$, where $x_{k}$ denotes the index of the symbol from the following set of possible indices $\boldsymbol{X}=\{0,1, \ldots, M-1\}$. Every symbol carries $l=\log _{2} M$ bits, using the appropriate mapping rule (natural, Gray, anti-Gray, etc.) The memory of the state is equal to $2 m+1$, with $2 m$ being the number of symbols that influence the observed symbol from both sides. The trellis has $M^{2 m+1}=64$ states $\left(\boldsymbol{s}_{0}, \boldsymbol{s}_{1}, \ldots, \boldsymbol{s}_{63}\right)$, each of which corresponds to a different 3 -symbol patterns (configurations). The state index is determined by considering $(2 m+1)$ symbols as digits in numerical system with the base $M$. For example, in Fig. 4, the quaternary numerical system (with the base 4) is used. The left column in dynamic trellis represents the current states and the right column denotes the terminal states. The branches are labeled by two symbols, the first symbol is an input symbol (the blue symbol), and the output symbol is the central symbol of terminal state (the red symbol). For the complete description of the dynamical trellis, the transition PDFs $p\left(y_{j} \mid x_{j}\right)=p\left(y_{j} \mid \boldsymbol{s}\right), \boldsymbol{s} \in \boldsymbol{S}$ are needed; where $\boldsymbol{S}$ is the set of states in the trellis. The conditional PDFs can be determined by using instanton-Edgeworth expansion method we proposed in Ivkovic et al. ${ }^{10}$. The number of edges originating in any of the left-column states is $M$, and the number of merging edges in arbitrary terminal state is also $M$.

The information rate can be calculated, as already introduced in (7), by:

$$
I(\mathbf{Y} ; \mathbf{X})=H(\mathbf{Y})-H(\mathbf{Y} \mid \mathbf{X}),
$$

where $H(\boldsymbol{U})=\mathrm{E}\left(\log _{2} P(\boldsymbol{U})\right)$ denotes the entropy of a random variable $\boldsymbol{U}$ and $\mathrm{E}(\cdot)$ denotes the mathematical expectation operator. By using the Shannon-McMillan-Brieman theorem that states ${ }^{11}$ :

$$
E\left(\log _{2} P(\boldsymbol{Y})\right)=\lim _{n \rightarrow \infty}(1 / n) \log _{2} P(\boldsymbol{y}[1, n]),
$$

the information rate can be determined by calculating $\log _{2}(P(y[1, n]))$, by propagating the sufficiently long source sequence. By substituting Eq. (14) into Eq. (13) we obtain the following expression suitable for practical calculation of IID channel capacity 


$$
I(\boldsymbol{Y} ; \boldsymbol{X})=\lim _{n \rightarrow \infty} \frac{1}{n}\left[\sum_{i=1}^{n} \log _{2} P\left(y_{i} \mid \boldsymbol{y}[1, i-1], \boldsymbol{x}[1, n]\right)-\sum_{i=1}^{n} \log _{2} P\left(y_{i} \mid \boldsymbol{y}[1, i-1]\right)\right] .
$$

The first term in (15) can be straightforwardly calculated from conditional PDFs because $P\left(y_{i} \mid \mathbf{y}[1, \mathrm{i}-1], x[1, n]\right)=P\left(y_{i} \mid \boldsymbol{x}[i-\right.$ $m, i+m])=P\left(y_{i} \mid \boldsymbol{s}\right)$. To calculate $\log _{2} P\left(y_{i} \mid \boldsymbol{y}[1, i-1]\right)$ we use the forward recursion of the multilevel BCJR algorithm ${ }^{9}$, wherein the forward metric $\alpha_{j}(\boldsymbol{s})=\log \left\{p\left(\boldsymbol{s}_{j}=\boldsymbol{s}, \boldsymbol{y}[1, j]\right)\right\}(j=1,2, \ldots, n)$, and the branch metric $\gamma_{j}\left(\boldsymbol{s}^{\prime}, \boldsymbol{s}\right)=\log \left[p\left(\boldsymbol{s}_{j}=\boldsymbol{s}, y_{j}, \boldsymbol{s}_{j-1}=\boldsymbol{s}^{\prime}\right)\right]$ are defined as follows:

$$
\begin{gathered}
\alpha_{j}(\boldsymbol{s})=\underset{\boldsymbol{s}^{\prime}}{\max ^{\prime}} *\left[\alpha_{j-1}\left(\boldsymbol{s}^{\prime}\right)+\gamma_{j}\left(\boldsymbol{s}^{\prime}, \boldsymbol{s}\right)-\log _{2} M\right] \\
\gamma_{j}\left(\boldsymbol{s}^{\prime}, \boldsymbol{s}\right)=\log \left[p\left(y_{j} \mid \boldsymbol{x}[j-m, j+m]\right)\right],
\end{gathered}
$$

where the max ${ }^{*}$-operator is defined by $\max ^{*}(x, y)=\log \left(\mathrm{e}^{x}+\mathrm{e}^{y}\right)=\max (x, y)+\log [1+\exp (-|x-y|)]$. The $i$ th term $\log _{2} P\left(y_{i} \mid y[1, i-1]\right)$ can be calculated iteratively by

$$
\log _{2} P\left(y_{i} \mid \boldsymbol{y}[1, i-1]\right)=\max _{\boldsymbol{s}}^{*} \alpha_{i}(\boldsymbol{s}),
$$

where $\max ^{*}$-operator was applied for all $\boldsymbol{s} \in \boldsymbol{S}$ ( $\boldsymbol{S}$ denotes the set of states in the trellis shown in Fig. 4(a)). An example of forward recursion step for 4-level modulation format (QPSK) is shown in Fig. 4(b), where $s$ denotes an arbitrary terminal state, which has $M=4$ edges originating from corresponding initial states, denoted as $\boldsymbol{s}_{1}{ }_{1}, \boldsymbol{s}_{2}{ }_{2}, \boldsymbol{s}_{3}{ }_{3}$, and $\boldsymbol{s}_{4}{ }_{4}$. The forward metric of state $\boldsymbol{s}$ in $j$ th step $(j=1,2, \ldots, n)$ is updated by preserving the maximum term (in max ${ }^{*}$-sense) $\alpha_{j-1}\left(\boldsymbol{s}_{\mathrm{k}}{ }_{\mathrm{k}}\right)+\gamma_{j}\left(\boldsymbol{s}_{,}, \boldsymbol{s}_{k}\right)$ $(k=1,2,3,4)$. The procedure is repeated for every state in column of terminal states of $j$ th step $(j=1,2, \ldots, n)$.

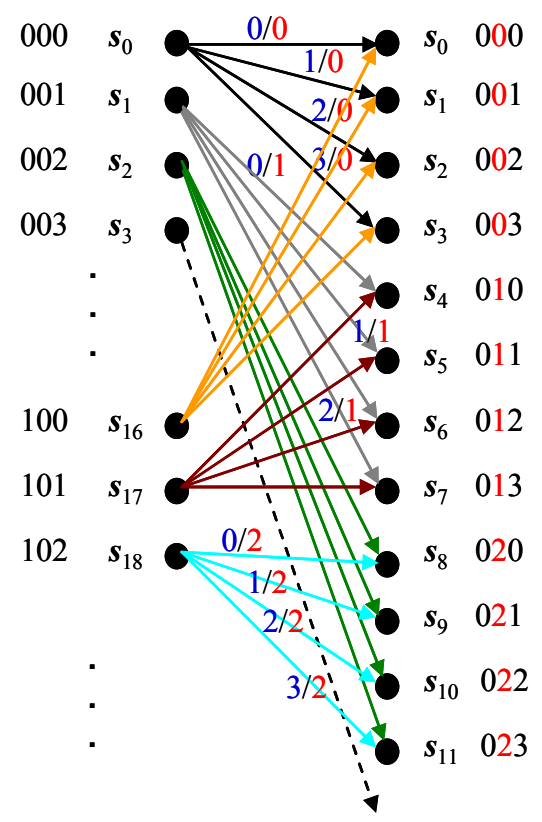

(a)

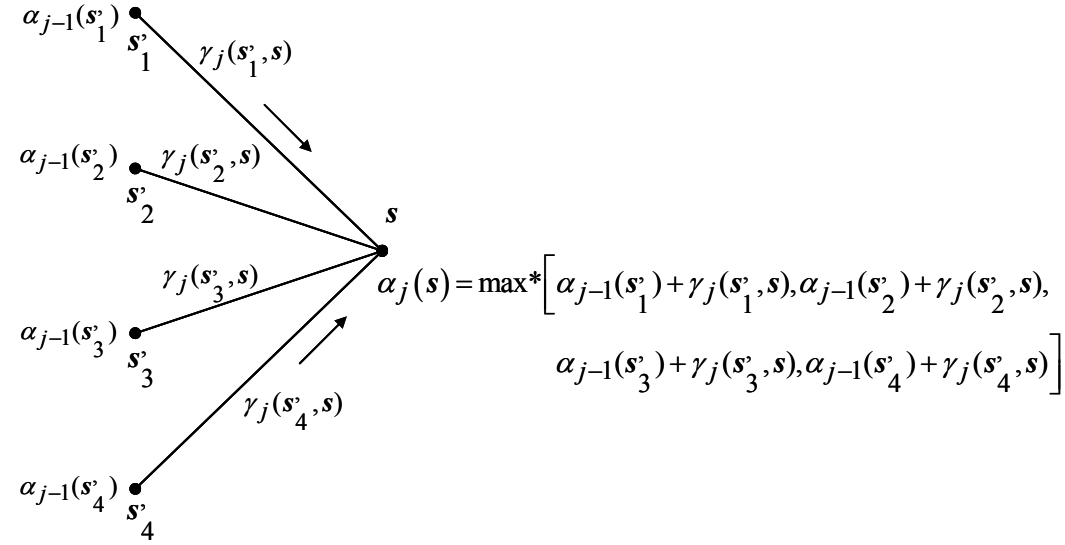

(b)

Figure 4. (a) Trellis description of fiber-optics channel, and (b) the forward recursion step for $M=4$-level BCJR equalizer.

In Fig. 5 we show the IID channel capacity against the number of spans, for dispersion map shown in Fig. 6 and QPSK modulation format of aggregate data rate $100 \mathrm{~Gb} / \mathrm{s}$, for two different memories in trellis description of the channel. The span length is set to $L=120 \mathrm{~km}$, and each span consists of $2 L / 3 \mathrm{~km}$ of $\mathrm{D}_{+}$fiber followed by $L / 3 \mathrm{~km}$ of $\mathrm{D}_{\text {- fiber, with pre- }}$ compensation of $-1600 \mathrm{ps} / \mathrm{nm}$ and corresponding post-compensation. The dispersion map is composed of periodically

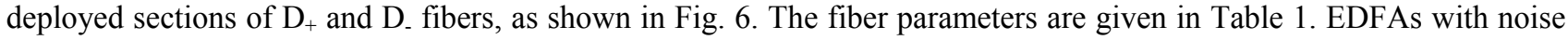
figure of $5 \mathrm{~dB}$ are deployed after every fiber section, the bandwidth of optical filter is set to $3 R_{s}$ and the bandwidth of 
electrical filter to $0.7 R_{s}$, with $R_{s}$ being the symbol rate that was set to $50 \mathrm{Giga}$ symbols $/ \mathrm{s}$. We see that the total transmission distance for trellis memory $m=1$ and channel code of code rate $R=0.8$ is $9600 \mathrm{~km}$, which is $2400 \mathrm{~km}$ better than that for trellis memory $m=0$ (i.e., the memoryless case).

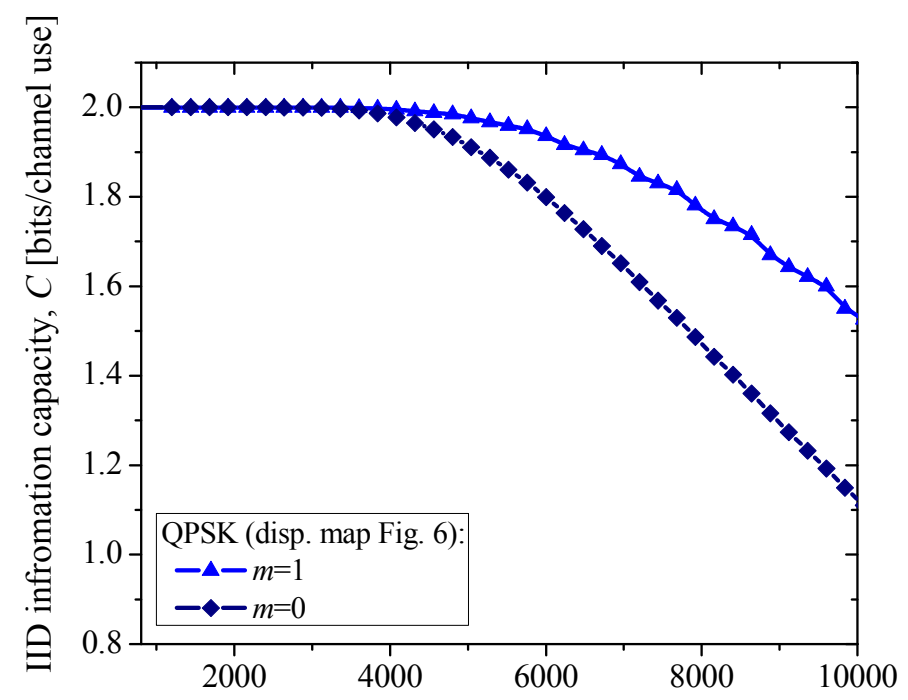

Total transmission distance, $L[\mathrm{~km}]$

Figure 5. IID information capacity for QPSK and 8PSK of symbol rate $50 \mathrm{GS} / \mathrm{s}$ against the transmission distance.

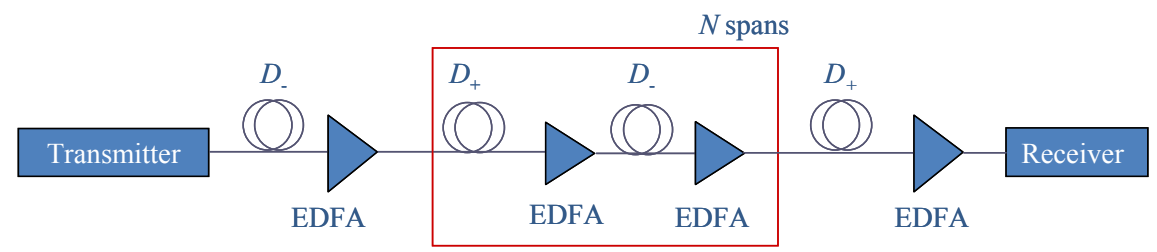

Figure 6. Dispersion map under consideration.

Table 1. Fiber parameters.

\begin{tabular}{|c|c|c|}
\hline Parameters & D $_{+}$FIBER & D. FIBER \\
\hline Dispersion $[\mathrm{ps} /(\mathrm{nm} \mathrm{km})]$ & 20 & -40 \\
\hline Dispersion Slope $\left[\mathrm{ps} /\left(\mathrm{nm}^{2} \mathrm{~km}\right)\right]$ & 0.06 & -0.12 \\
\hline Effective Cross-sectional Area $\left[\mu \mathrm{m}^{2}\right]$ & 110 & 50 \\
\hline Nonlinear Refractive Index $\left[\mathrm{m}^{2} / \mathrm{W}\right]$ & $2.6 \cdot 10^{-20}$ & $2.6 \cdot 10^{-20}$ \\
\hline Attenuation Coefficient $[\mathrm{dB} / \mathrm{km}]$ & 0.19 & 0.25 \\
\hline
\end{tabular}

\section{LDPC-CODED MULTILEVEL TURBO EQUALIZATION}

We further describe a channel capacity approaching coding scheme. The proposed scheme, shown in Fig. 7, is based on multilevel $(M>2)$ maximum a posteriori probability (MAP) turbo equalization. It is composed of two ingredients: (i) the multilevel BCJR algorithm based equalizer, and (ii) the LDPC decoder. The BCJR equalizer operates on trellis channel description given above (see Fig. 4(a)), and provides soft symbol log-likelihood ratios (LLRs) used in LDPC decoding 
process. The channel codes are based on large girth LDPC $\operatorname{codes}^{7,14}$. The use of large girth codes increases the minimum distance, and de-correlates the extrinsic info in LDPC decoding process. The extrinsic information transfer (EXIT) chart approach due to ten Brink ${ }^{12}$ is used to match the LDPC decoders, for large-girth quasi-cyclic LDPC codes, and multilevel BCJR equalizer.

The results of simulations for a single-channel optical QPSK transmission system operating at 50 Giga symbols/s, with dispersion map described above, are shown in Fig. 8. The number of spans was changed from 4 to 84, the uncoded BER at 50 Giga symbols/s and BER after LDPC decoding at line rate $R_{\mathrm{l}}=R_{\mathrm{s}} / R\left(R_{\mathrm{s}}=50\right.$ Giga symbols $/ \mathrm{s}, R$ is the code rate $)$ were calculated and given in Fig. 8 against the number of spans. We can see that for 22 spans 4-evel BCJR equalizer with memory $m=1$ provides more than one order in magnitude improvement in BER over memoryless case $(m=0)$. For the multilevel turbo equalization scheme based on 4-level BCJR equalizer of memory $m=0$ and the LDPC $(16935,13550)$ code of girth-10 and column weight 3, we achieve transmission over 55 spans $(6600 \mathrm{~km})$ without any error. On the other hand, for the turbo equalization scheme based on 4-level BCJR equalizer of memory $m=1$ and the same LDPC code, we are able to achieve $8160 \mathrm{~km}$ of error free transmission at aggregate rate of $100 \mathrm{~Gb} / \mathrm{s}$. To achieve the channel capacity (9600 km) with code rate $R=0.8$, the girth-12 LDPC codes of length above 100,000 are needed.

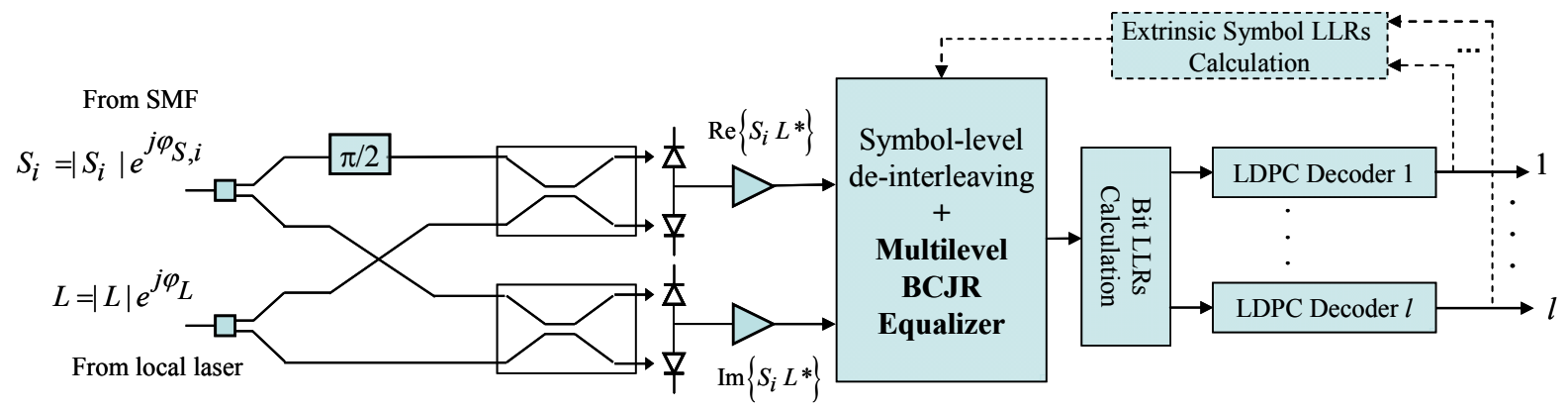

Figure 7. Proposed multilevel turbo equalization scheme.

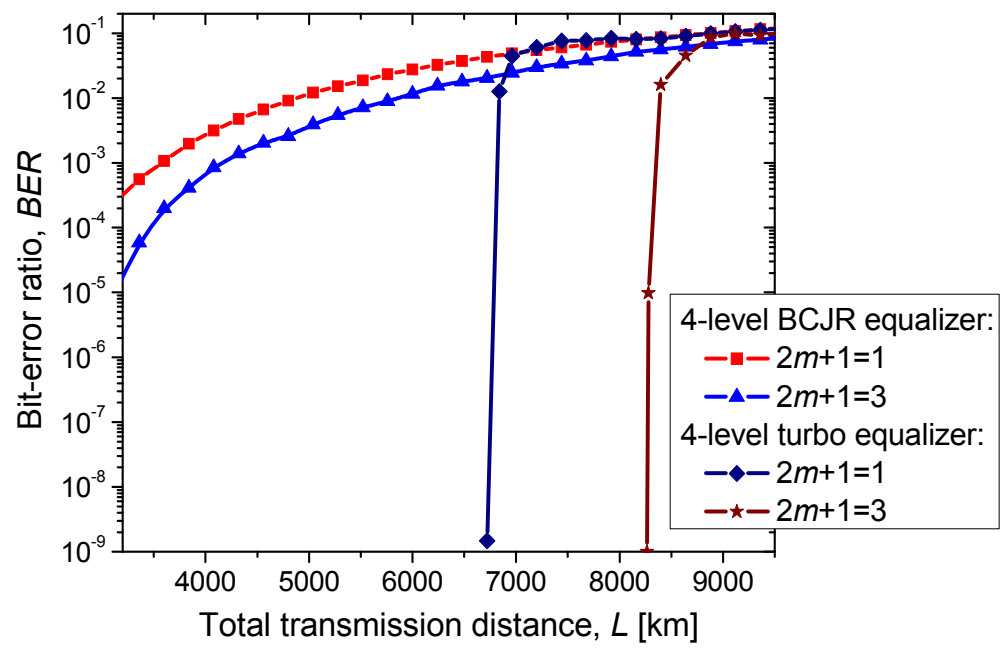

Figure 8. BER performance of 4-level turbo equalizer.

\section{CONCLUSION}

In this paper, we described a method to determine the channel capacity of arbitrary multilevel modulation scheme by modeling the fiber-optic channel as dynamical nonlinear ISI channel with memory. In addition, we proposed a multilevel turbo-equalization scheme that is able closely to approach the channel capacity. This method is universal and applicable to arbitrary multilevel modulation with both direct detection and coherent detection. This scheme can be used to simultaneously compensate for fiber nonlinearities, PMD and chromatic dispersion. We demonstrated by Monte Carlo simulations that this scheme is efficient in compensation of fiber nonlinearities. We have shown the even with small memory assumption we are able to achieve $100 \mathrm{~Gb} / \mathrm{s}$ per DWDM channel transmission over $9600 \mathrm{~km}$. We showed that 
with this scheme we can straightforwardly upgrade currently installed $10 \mathrm{~Gb} / \mathrm{s}$ optical transmission systems to $100 \mathrm{~Gb} / \mathrm{s}$. In our recent paper ${ }^{16}$, we have shown that similar scheme can be used to compensate for PMD and any imbalance in phase between I and Q channels.

\section{REFERENCES}

1. Narimanov, E. E. and Mitra, P., "The channel capacity of a fiber optics communication system: perturbation theory," IEEE/OSA J. Lightwave Technol., 20(3), 530-537(2002).

2. Narimanov, E. and Patel, P., "Channel capacity of fiber optics communications systems: WDM vs. TDM," in Proc. Conf. on Lasers and Electro-Optics (CLEO '03), 2003, pp. 1666 - 1668.

3. Mitra, P. P. and Stark, J. B., "Nonlinear limits to the information capacity of optical fiber communications," Nature, 411, 1027-1030 (2001).

4. Turitsyn, K. S., Derevyanko, S. A., Yurkevich, I. V. and Turitsyn, S. K., "Information capacity of optical fiber channels with zero average dispersion,” Phys. Rev. Lett., 91 (20), 203901 (2003).

5. Essiambre, R.-J., Foschini, G. J., Kramer, G. and Winzer, P. J., "Capacity limits of information transport in fiberoptic networks," Phys. Rev. Lett. 101, 163901-1 - 163901-4 (2008).

6. Djordjevic, I. B., Vasic, B., Ivkovic, M. and Gabitov, I., "Achievable information rates for high-speed long-haul optical transmission," IEEE/OSA J. Lightw. Technol. 23(11), 3755-3763 (2005).

7. I. B. Djordjevic, L. L. Minkov, and H. G. Batshon, "Mitigation of linear and nonlinear impairments in high-speed optical networks by using LDPC-coded turbo equalization,” IEEE J. Sel. Areas Comm. 26(6), 73-83 (2008).

8. Ivkovic, M., Djordjevic, I. B. and Vasic, B., "Calculation of achievable information rates of long-haul optical transmission systems using instanton approach,” IEEE/OSA J. Lightw. Technol., 25(5), 1163-1168 (2007).

9. L. R. Bahl, J. Cocke, F. Jelinek, and J. Raviv, "Optimal decoding of linear codes for minimizing symbol error rate," IEEE Trans. Inform. Theory IT-20(3), 284-287 (1974).

10. Ivkovic, M., Djordjevic, I., Rajkovic, P. and Vasic, B., "Pulse energy probability density functions for long-haul optical fiber transmission systems by using instantons and Edgeworth expansion," IEEE Photon. Technol. Lett. 19(20), 1604 - 1606 (2007).

11. Cover, T. M. and Thomas, J. A., [Elements of Information Theory]. New York: Wiley, 1991.

12. Ingels, F. M., [Information and Coding Theory]. Scranton: Intext Educational Publishers, 1971.

13. Chung, S., et al., "On the design of low-density parity-check codes within $0.0045 \mathrm{~dB}$ of the Shannon Limit," IEEE Comm. Lett., 5(2), 58-60 (2001).

14. Fossorier, M. P. C., "Quasi-cyclic low-density parity-check codes from circulant permutation matricies," IEEE Trans. Inform. Theory, 50(8), 1788-1794 (2004).

15. ten Brink, S., "Convergence behavior of iteratively decoded parallel concatenated codes," IEEE Trans. Comm. 40(10), 1727-1737 (2001).

16. Minkov, L. L., Djordjevic, I. B., Xu, L., and Wang, T., "PMD compensation in polarization multiplexed multilevel modulations by turbo equalization," IEEE Photon. Technol. Lett., accepted for publication. 